\title{
Value of MRI and diffusion-weighted MRI for the diagnosis of locally recurrent rectal cancer
}

\author{
Doenja M. J. Lambregts • Vincent C. Cappendijk • \\ Monique Maas • Geerard L. Beets • \\ Regina G. H. Beets-Tan
}

Received: 14 October 2010 /Revised: 22 November 2010 / Accepted: 25 November 2010 /Published online: 16 January 2011

(C) The Author(s) 2011. This article is published with open access at Springerlink.com

\begin{abstract}
Objectives To evaluate the accuracy of standard MRI, diffusion-weighted MRI (DWI) and fusion images for the diagnosis of locally recurrent rectal cancer in patients with a clinical suspicion of recurrence.

Methods Forty-two patients with a clinical suspicion of recurrence underwent 1.5-T MRI consisting of standard T2weighted FSE (3 planes) and an axial DWI (b0,500,1000). Two readers $(R 1, R 2)$ independently scored the likelihood of recurrence; [1] on standard MRI, [2] on standard MRI+DWI, and [3] on T2-weighted+DWI fusion images.

Results 19/42 patients had a local recurrence. R1 achieved an area under the ROC-curve (AUC) of 0.99 , sensitivity $100 \%$ and specificity $83 \%$ on standard MRI versus $0.98,100 \%$ and $91 \%$ after addition of DWI $(p=0.78)$. For R2 these figures were $0.87,84 \%$ and $74 \%$ on standard MRI and $0.91,89 \%$ and $83 \%$ with DWI ( $p=0.09)$. Fusion images did not significantly improve the performance. Interobserver agreement was $\mathrm{k} 0.69$ for standard MRI, $\mathrm{k} 0.82$ for standard MRI+DWI and $\mathrm{k} 0.84$ for the fusion images.

Conclusions MRI is accurate for the diagnosis of locally recurrent rectal cancer in patients with a clinical suspicion of recurrence. Addition of DWI does not significantly improve its performance. However, with DWI specificity and interobserver agreement increase. Fusion images do not improve accuracy.
\end{abstract}

D. M. J. Lambregts · V. C. Cappendijk · M. Maas •

R. G. H. Beets-Tan $(\bowtie)$

Department of Radiology, Maastricht University Medical Centre,

P.O. Box 5800, 6202 AZ, Maastricht, The Netherlands

e-mail: r.beets.tan@mumc.nl

D. M. J. Lambregts $\cdot$ M. Maas $\cdot$ G. L. Beets

Department of Surgery, Maastricht University Medical Centre,

P.O. Box 5800, 6202 AZ, Maastricht, The Netherlands
Keywords MRI · Diffusion magnetic resonance imaging · Rectal neoplasms . Local neoplasm recurrence $\cdot$ Diagnosis

\section{Introduction}

Around $35 \%$ of colorectal cancer patients who undergo curative surgical treatment will develop recurrent disease, the majority of which occurring during the first 3 years after surgery [1]. Relapse mainly occurs in the liver $(20 \%)$ and lungs $(5-10 \%)$, while approximately $5-15 \%$ of patients will develop a locoregional recurrence [2]. These local recurrences are associated with significant morbidity and impaired quality of life. Whereas locally recurrent rectal cancer has long been regarded as a rarely curable disease, patients are now increasingly being considered for curative salvage surgery, often combined with (neo)adjuvant chemo and/or radiation treatment. The chances of cure after resection are highest when all resection margins are microscopically free of tumour [3]. To increase the chance of cure it is therefore crucial to diagnose local recurrences when still in an early stage. At present, it is not clear whether imaging is beneficial during the surveillance of patients after rectal cancer surgery and trials to establish the role of imaging are ongoing [4-7]. If, however, during surveillance a patient is suspected of a local recurrence based on clinical symptoms and/or rising carcinoembryonic antigen (CEA) levels, CT is the first investigation of choice to confirm or rule out the presence of a local or distant relapse $[4,7,8]$. A pelvic mass that enlarges on consecutive postoperative CT studies is highly suspicious for a local recurrence, although the diagnosis is not always easy to make $[9,10]$. Often the CT findings are equivocal and in those cases ${ }^{18} \mathrm{~F}$-Fluorodeoxygenase Positron Emission Tomography (FDG-PET) or combined PET/CT have 
proven beneficial in identifying local tumour re-growth [10, 11]. False positive FDG-PET findings may, however, occur in areas of postoperative inflammatory scar tissue, in the case of displaced small bowel loops and because of the proximity of the urinary bladder. Moreover, FDG-PET lacks accuracy in the evaluation of a recurrence of the mucinous tumour type and is less useful to evaluate tumour resectability due to its insufficient spatial resolution [11, 12]. MRI on the other hand has proven valuable for assessing the resectability of a diagnosed local recurrence of rectal cancer [13], but its role in the detection of recurrent tumour is not well established. The main difficulty with MRI is the identification of a small growing tumour in an area of fibrotic scar tissue [14-16]. Diffusionweighted MR imaging (DWI) is a promising technique for the detection of small tumour volumes and its benefit has also been shown in pelvic tumours [17-19]. The role of DWI in the detection of locally recurrent rectal cancer has, however, not been investigated yet. As an addition to standard anatomical MRI, DWI could be useful to increase the diagnostic performance for detection of locoregional tumour recurrences [20]. Furthermore, previous studies have suggested that image fusion of anatomical MRI and DWI can contribute to a better detection of both primary malignancies and recurrent disease, though again its role in rectal cancer is not clear $[20,21]$.

The aim of this study therefore is to evaluate the accuracy of standard pelvic MRI for the diagnosis of locoregional tumour recurrence in patients with a clinical suspicion of a recurrence after curative rectal cancer surgery and to evaluate whether the addition of DWI and/or image fusion of standard MRI and DWI improves its performance.

\section{Materials and methods}

\section{Patients}

This study retrospectively evaluated 42 consecutive patients who underwent MR imaging for the evaluation of the presence of a locally recurrent rectal cancer between June 2007 and April 2010. Because of the retrospective nature of the study, informed consent was waived. The baseline patient characteristics are given in Table 1. Twenty-six patients were male and 16 were female. Median age was 66 years (range 22-87). All patients had previously undergone curative-intent surgical treatment for primary rectal cancer. Patients were referred for MRI when during follow-up there was a clinical suspicion for a locoregional tumour recurrence based on symptoms (pain, faecal blood loss, changed bowel habits), clinical examination, increased serum CEA and/or the presence of metachronous distant tumour lesions. Eleven patients first underwent a CT examination, before being referred for MRI. Nineteen patients were diagnosed with a locally recurrent rectal tumour, while in 23 patients no evidence of recurrence was found. The locations of recurrence were as follows: anastomotic $(n=2)$, presacral $(n=3)$, lateral pelvic nodes $(n=3)$, pelvic floor $(n=3)$, local excision scar $(n=5)$, rectal lumen $(n=1)$, peri-prostatic $(n=1)$, and gluteus muscle $(n=$ 1). The median time interval between surgical treatment of the primary rectal tumour and the follow-up MRI for local recurrence evaluation was 40 months (range 3-320). Median follow-up between the MRI and present was 20 months (range 4-42).

\section{MR imaging}

Patients did not receive bowel preparation or spasmolytics. MR imaging was performed at 1.5-T (Intera; Philips Medical Systems, Best, The Netherlands). For signal reception, a phased-array body coil was used. The standard rectal MRI protocol consisted of 2D T2-weighted (T2W) fast spin-echo (FSE) sequences in three planes; sagittal, axial and coronal (TR/TE $8456 / 130 \mathrm{msec}, 90^{\circ}$ flip angle, 25 echotrain length, $6 \mathrm{NSA}, 0.78 \times 1.14 \times 3.00 \mathrm{~mm}$ acquisition voxel size, 30 slices, $6.03 \mathrm{~min}$ acquisition time). Since 2006, a DWI sequence had been implemented as part of the standard rectal MRI protocol. The DWI sequence was an axial diffusion-weighted sequence with background body signal suppression (DWIBS [22], b-values 0,500,1000 s/ $\mathrm{mm}^{2}, \mathrm{TR} /$ TE $4829 / 70 \mathrm{msec}$, EPI factor $53,4 \mathrm{NSA}, 2.50 \times 3.11 \times$ $5.00 \mathrm{~mm}$ acquisition voxel size, 50 slices, $10.37 \mathrm{~min}$ acquisition time). The axial T2-weighted and DWI sequences were obtained in identical planes. These were routinely angled perpendicular to the rectal lumen. When no remaining rectal lumen was present (after abdominoperineal or extended resection) or in case of a pelvic mass located outside the rectal lumen, the axial sequences were angled perpendicular to the interface between the local area of suspicion and the closest structure or organ. ADC maps including all three b-values were automatically generated in greyscale at the operating system.

\section{Image fusion of T2-weighted MRI and DWI}

MR images were exported in DICOM format and transferred to a Macintosh computer (iMac, Apple, Cupertino, CA, USA). Fusion images of the axial T2-weighted and b1000 diffusion-weighted images were generated using OsiriX Medical Imaging Software, an open-source postprocessing software tool [23]. The field of view and slice level of the diffusion images were automatically adjusted to exactly match the T2-weighted MRI. The DWI images were then converted to a red-to-yellow colour scale and overlaid on the $\mathrm{T} 2$-weighted images. 
Table 1 Baseline characteristics of study patients

\begin{tabular}{|c|c|c|c|}
\hline \multirow[t]{2}{*}{ Variable } & \multicolumn{3}{|l|}{$\mathrm{N}^{\mathrm{o}}$ Patients } \\
\hline & Recurrence $(n=19)$ & No Recurrence $(n=23)$ & Total $(n=42)$ \\
\hline \multicolumn{4}{|l|}{ Sex } \\
\hline Male & 13 & 13 & 26 \\
\hline Female & 6 & 10 & 16 \\
\hline Median age & 68 (range 35-87) & 64 (range $22-81$ ) & 66 (range $22-87$ ) \\
\hline \multicolumn{4}{|l|}{ Treatment of primary tumour } \\
\hline Surgery & 6 & 3 & 9 \\
\hline $5 \times 5$ Gy+surgery & 2 & 5 & 7 \\
\hline Neoadjuvant and/or adjuvant chemoradiation+surgery & 11 & 15 & 26 \\
\hline \multicolumn{4}{|l|}{ Type of primary surgery } \\
\hline Total mesorectal excision & 14 & 22 & 36 \\
\hline Local excision (transanal endoscopic microsurgery) & 5 & 1 & 6 \\
\hline \multicolumn{4}{|l|}{ Radicality of primary resection } \\
\hline $\mathrm{R} 0$ & 14 & 19 & 33 \\
\hline $\mathrm{R} 1$ & 3 & 4 & 7 \\
\hline $\mathrm{R} 2$ & 2 & 0 & 2 \\
\hline \multicolumn{4}{|l|}{ Synchronous metastases } \\
\hline Liver & 5 & 4 & 9 \\
\hline Lung & 1 & 1 & 2 \\
\hline Other & 0 & 0 & 0 \\
\hline \multicolumn{4}{|l|}{ Metachronous metastases } \\
\hline Liver & 4 & 4 & 8 \\
\hline Lung & 5 & 5 & 10 \\
\hline Other & 4 & 2 & 6 \\
\hline Median CEA at time of MRI $(\mu \mathrm{g} / \mathrm{l})$ & 8 (range 1-2154) & 3 (range $0,5-55$ ) & 4 (range $0,5-2154$ ) \\
\hline Previous recurrence & 4 & 2 & 6 \\
\hline
\end{tabular}

\section{Image evaluation}

The MR images were independently analysed by two readers from an expert referral center: a pelvic MR expert with 13 years expertise in reading MRI of rectal cancer (RGHBT) and a junior GI radiologist with 3 years specific expertise in pelvic MRI (VCC). The readers were aware of the treatment, histological stage and tumour type of the primary rectal cancer, but were blinded to each other's scoring results and to whether or not previous CT imaging for the recurrence was present. They were also blinded to the results from endoscopy, other imaging, surgery and histopathology. This study design was chosen to reproduce the clinical standard of practice, where radiologists are exposed to a patient's history of the primary tumour. Image evaluation consisted of three separate reading protocols conducted within the same reading session: (a) the readers first evaluated the T2-weighted FSE images only (further referred to as 'standard MRI'), (b) immediately thereafter the scoring was repeated after the b1000 diffusion images were added to the standard MRI (further referred to as 'standard MRI+DWI'). The low b-value diffusion images and the ADC map were also at the readers' disposal. Finally (c) the fusion images of T2weighted MRI+DWI were assessed. Patients were evaluated in random order. The readers scored the likelihood of a local tumour recurrence using a 5-point confidence level scale $(0=$ definitely absent, $1=$ probably absent, $2=$ possibly absent/present, $3=$ probably present, $4=$ definitely present). On T2-weighted MRI, focal areas showing a mass effect, a signal intensity isointense to muscle and/or a nodular or infiltrative border were considered highly suspicious for tumour recurrence. On the high b-value (b1000) DWI, focal areas of high signal intensity, corresponding with possible tumour locations on standard MRI were considered a criterion for tumour recurrence (Fig. 1). On the fusion images focal areas of hyperintensity (yellow), corresponding with possible tumour on T2-weighted MRI were considered suspicious for recurrent tumour. When a recurrent tumour was suspected, the largest tumour diameter was measured on T2-weighted MRI. 

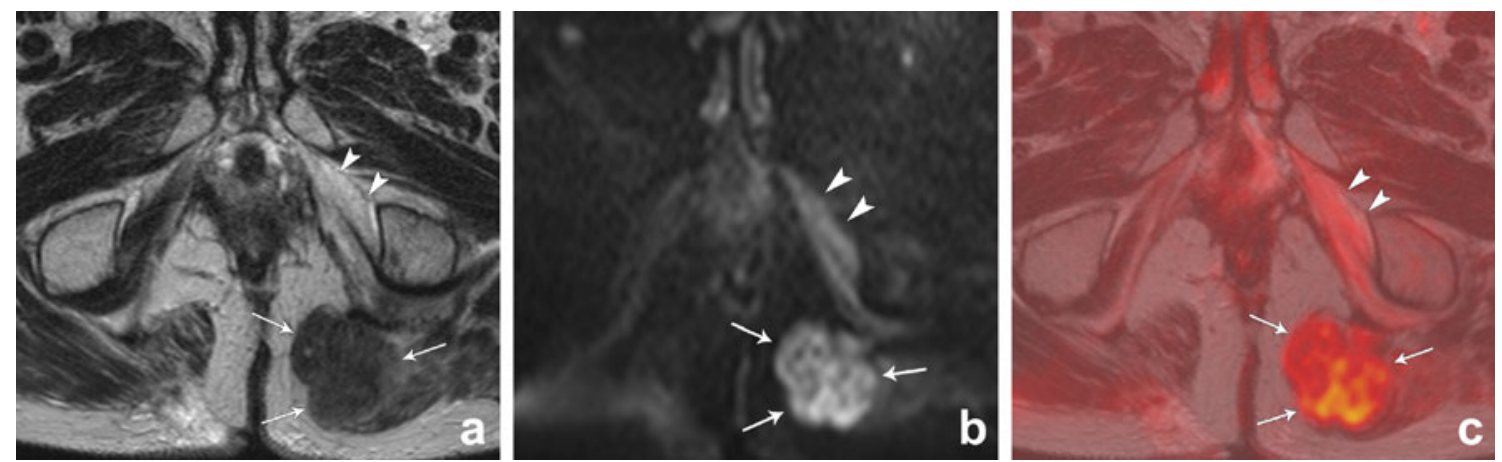

Fig. 1 T2-weighted, b1000 diffusion-weighted and T2W+DWI fusion images of a male patient with a local tumour recurrence situated in the left gluteus muscle (arrows). On T2-weighted MRI (a), the signal of the tumour is isointense compared to the muscles. On the DWI (b) and fusion images (c) the tumour is visible as a lesion with high signal intensity, compared to the suppressed signal of the surrounding structures. Note the necrotic changes in the left internal obturator muscle (arrowheads), resulting in slightly increased signal intensity on the DWI and fusion images

\section{Standard reference}

The presence of a local recurrence $(n=19)$ was histologically confirmed in 16 patients (surgical resection in 11 and positive biopsy of the suspected tumour area in 5 patients). In 3 patients the diagnosis of a local recurrence was made based on concordant positive FDG-PET/CT findings (i.e. positive FDG uptake at the exact location suspected for tumour on MRI) as well as follow-up MRI and/or FDGPET/CT examinations. Furthermore, an inguinal lymph node metastasis was confirmed by biopsy in 1 patient. These 3 patients did not undergo surgical treatment of their recurrence, because of unresectable disease. The absence of a local recurrence $(n=23)$ was confirmed by a combination of the following: no abnormal findings on corresponding FDG/PET-CT $(n=8)$, no abnormal findings on colonoscopy and/or negative biopsy results $(n=4)$ and/or no signs of tumour recurrence on consecutive imaging examinations (CT, MRI and/or FDG-PET/CT) during a follow-up period of at least 1 year $(n=22)$. Median follow-up time for the patients without recurrence was 23 months (range 14-41).

\section{Statistical analysis}

Statistical analyses were performed using the Statistical Package for the Social Sciences (SPSS, version 16.0, Inc., Chicago, IL). Receiver operator characteristic (ROC) curve analyses were performed to evaluate the diagnostic performance for (a) standard MRI only, (b) standard MRI+DWI and (c) fused T2-weighted+DWI images. Corresponding areas under the ROC-curve (AUC), sensitivities, specificities, positive predictive values (PPV) and negative predictive values (NPV) with $95 \%$ confidence intervals (CI) were calculated. For these analyses it had been decided at the start of the study to dichotomise the confidence level scores between 1 (probably absent) and 2 (possibly absent/present). Differences in diag- nostic performance between the 3 reading strategies were analysed by comparing the ROC-curves according to the method described by DeLong et al [24]. Weighted kappavalues with quadratic kappa weighting $(0.00-0.20$ poor, $0.21-$ 0.40 fair, $0.41-0.60$ moderate, $0.61-0.80$ good and $0.81-1.00$ excellent interobserver agreement) were calculated to determine interobserver agreement between the two readers [25]. P-values $<0.05$ were considered statistically significant.

\section{Results}

Diagnostic predictive values including $95 \%$ confidence intervals are provided in Table 2 for the three reading methods. The ROC-curves are displayed in Fig. 2.

\section{Standard MRI}

When using only standard MRI, reader 1 achieved an AUC of 0.99 , sensitivity $100 \%$ and specificity $83 \%$. For reader 2 these figures were $0.87,84 \%$ and $74 \%$.

Added value of DWI and fusion images

After addition of DWI, reader 1 achieved an AUC of 0.98, sensitivity $100 \%$ and specificity $91 \%$. Compared to standard MRI (AUC 0.99), the difference in AUC was not significant $(p=0.78)$. For reader 2 , AUC improved from 0.87 on standard MRI to 0.91 after addition of DWI ( $p=$ 0.09 ). He achieved a sensitivity of $89 \%$ and specificity of $83 \%$. The added value of DWI is illustrated in Fig. 3, in which a false positive finding on T2-weighted MRI was corrected after addition of DWI. The addition of fusion images did not improve the diagnostic performance as compared to standard MRI+DWI ( $p=0.41$ for reader 1 and $p=1.00$ for reader 2). 
Table 2 Diagnostic performance for identification of a local tumour recurrence

\begin{tabular}{|c|c|c|c|c|c|c|}
\hline \multirow[t]{2}{*}{ Parameter } & \multicolumn{3}{|l|}{ Reader 1} & \multicolumn{3}{|l|}{ Reader 2} \\
\hline & Standard MRI & Standard MRI+DWI & Fusion & Standard MRI & Standard MRI+DWI & Fusion \\
\hline Sensitivity & $100(19 / 19)$ & $100(19 / 19)$ & $100(19 / 19)$ & $84(16 / 19)$ & $89(17 / 19)$ & $84(16 / 19)$ \\
\hline $95 \% \mathrm{CI}$ & $88-100$ & $89-100$ & $89-100$ & $68-94$ & $75-97$ & $69-93$ \\
\hline Specificity & $83(19 / 23)$ & $91(21 / 23)$ & $91(21 / 23)$ & $74(17 / 23)$ & $83(19 / 23)$ & $83(19 / 23)$ \\
\hline $95 \% \mathrm{CI}$ & $73-83$ & $82-91$ & $82-91$ & $61-82$ & $70-89$ & $70-90$ \\
\hline PPV & $83(19 / 23)$ & $90(19 / 21)$ & $90(19 / 21)$ & $71(15 / 21)$ & $80(16 / 20)$ & $79(15 / 19)$ \\
\hline $95 \% \mathrm{CI}$ & $73-83$ & $80-91$ & $80-91$ & $59-81$ & $68-88$ & $65-89$ \\
\hline NPV & $100(19 / 19)$ & $100(21 / 21)$ & $100(21 / 21)$ & $85(17 / 20)$ & $90(19 / 21)$ & $86(19 / 22)$ \\
\hline $95 \% \mathrm{CI}$ & $88-100$ & $90-100$ & $90-100$ & $70-94$ & $77-97$ & $73-94$ \\
\hline Accuracy & $90(38 / 42)$ & $95(40 / 42)$ & $95(40 / 42)$ & $79(33 / 42)$ & $86(36 / 42)$ & $83(35 / 42)$ \\
\hline $95 \% \mathrm{CI}$ & $79-91$ & $85-95$ & $85-95$ & $65-87$ & $72-92$ & $70-92$ \\
\hline AUC & 0.99 & 0.98 & 0.98 & 0.87 & 0.91 & 0.91 \\
\hline $95 \% \mathrm{CI}$ & $0.96-1.00$ & $0.94-1.00$ & $0.93-1.00$ & $0.75-0.98$ & $0.82-1.00$ & $0.82-1.00$ \\
\hline
\end{tabular}

$P P V$ positive predictive value. $N P V$ negative predictive value. $A U C$ area under the ROC curve. $95 \% C I 95 \%$ confidence interval. Numbers are percentages, absolute numbers are given in parentheses. Reader 1 is a senior GI radiologist with 13 years specific pelvic MR expertise. Reader 2 is a junior GI radiologist with 3 years pelvic MR expertise.

\section{Interobserver agreement}

Interobserver agreement for standard MRI was good ( $\mathrm{K}$ 0.69). After the addition of DWI, agreement was excellent ( $\mathrm{K} 0.82$ ). Agreement for the T2W+DWI fusion images was excellent ( $\kappa$ 0.84).

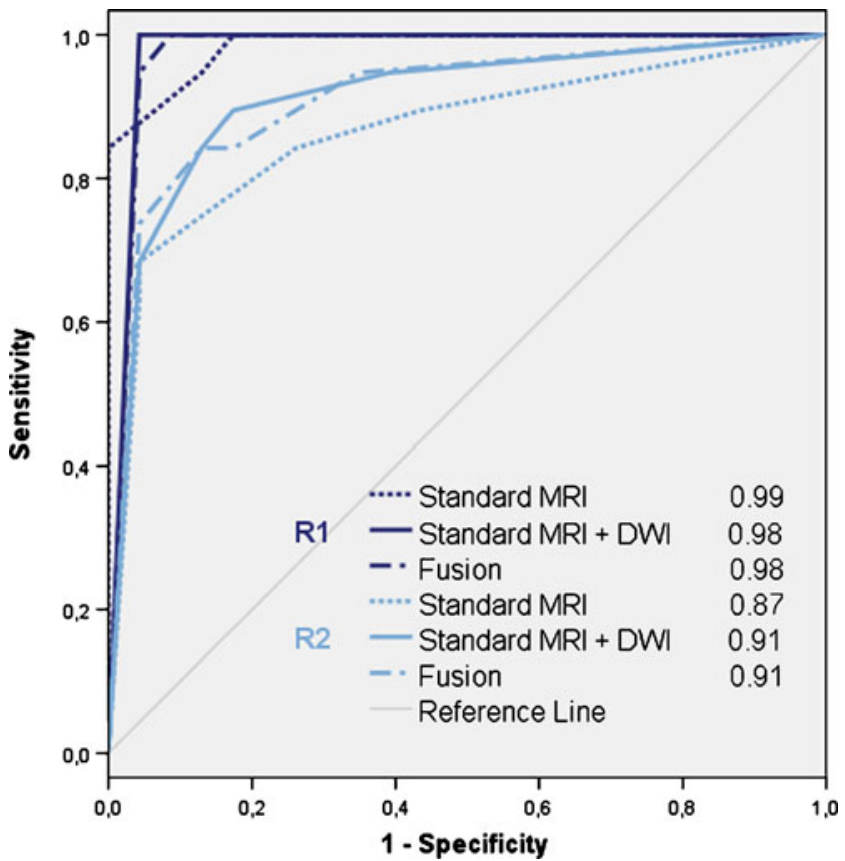

Fig. 2 Receiver operator characteristics curves and areas under the ROC-curve (AUC) for reader 1 (R1) and reader 2 (R2) for detection of locally recurrent rectal cancer by using (1) standard MRI, (2) standard MRI+DWI, and (3) T2-weighted MRI+DWI fusion images, respectively. There were no significant differences in AUC between the three reading designs for either of the two readers

\section{Lesion size}

In the 19 patients with recurrence, median lesion size (largest tumour diameter) was $37 \mathrm{~mm}$ (range 13-81 mm). Seven patients had a 'small' recurrence $(<20 \mathrm{~mm})$. Reader 1 detected all 7 small recurrences, both with standard MRI and after addition of DWI. Reader 2 missed one small recurrence on standard MRI, but detected all 7 after addition of DWI (Fig. 4).

Eleven patients had undergone CT imaging before MRI. In Table 3 and Fig. 5 the discrepancies between the CT findings and MRI scorings are illustrated.

\section{Discussion}

The aim of this study was to assess the performance of MRI for the diagnosis of locally recurrent rectal cancer in patients with a clinical suspicion of a recurrence after rectal cancer surgery and to evaluate whether the addition of diffusion-weighted imaging and/or image fusion of standard MRI and DWI improves its performance.

Our results indicate that standard MRI on its own has a high accuracy for the diagnosis of recurrent tumour in this specific group of patients. The addition of DWI does not significantly improve its performance. The high accuracy using only standard MRI could partly be due to the relatively high levels of expertise of the readers in this study. Furthermore, some patients had relatively large tumours, which were already correctly identified on morphological MR imaging. The benefit of DWI might be more pronounced for the detection of smaller tumours 

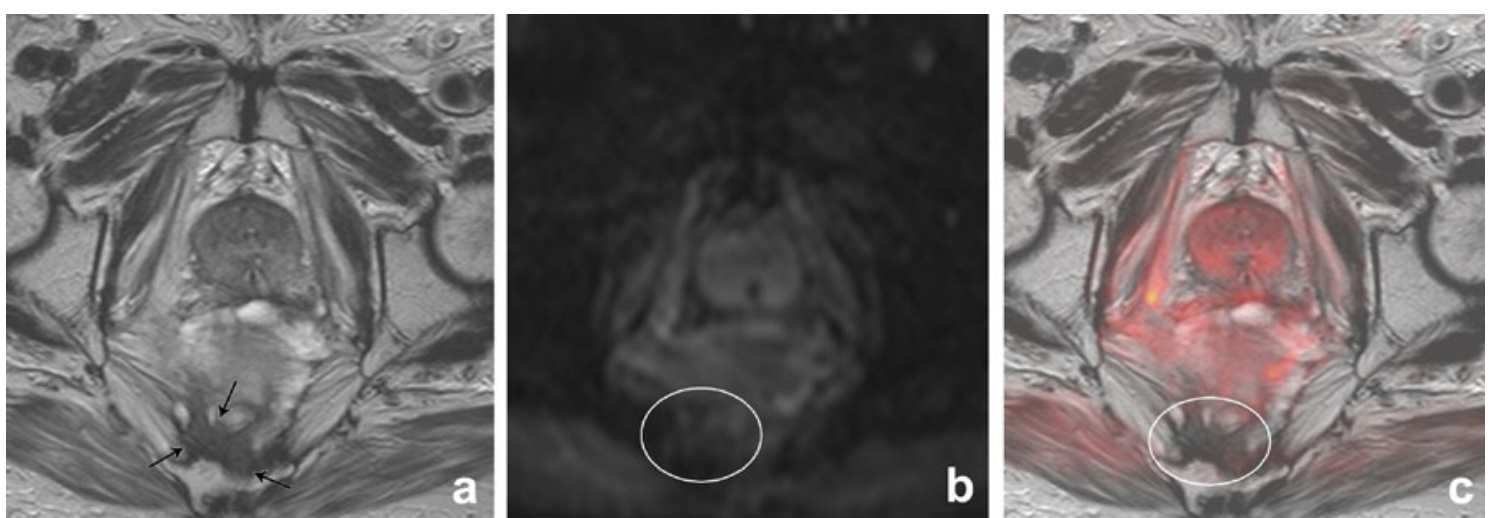

Fig. 3 Axial T2-weighted, b1000 diffusion-weighted and T2W+DWI fusion images of a male patient who had previously undergone a low anterior resection. On T2-weighted MRI (a) there is an area of intermediate signal intensity (arrows) that was erroneously interpreted

which are more difficult to detect within the fibrotic scar tissue. Results from a recent study by Syk et al. showed that mainly anastomotic recurrences - which tend to be smaller in size - are missed with imaging, again suggesting that the benefit from additional functional imaging may be higher in the detection of these small tumours [26]. In our study we indeed observed that DWI was helpful in such a case: the less experienced radiologist detected one of the small recurrences on MRI+DWI which he had overlooked before (Fig. 4). Larger patient studies are, however, required to further investigate and confirm the potential role of DWI in small tumours.

Of interest was the fact that adding DWI improved the interobserver agreement and reduced the number of false positives. DWI thus seems to increase the confidence of radiologists to rule out the presence of a recurrence (Fig. 3). This a relevant finding, as it is known from previous studies that standard MRI generally tends to overestimate the as a tumour recurrence by the first reader. On the DWI (b) and fusion images (c), there is no focal high signal intensity in this area (circle) and the reader adjusted his score. Follow-up imaging revealed no tumour recurrence presence of tumour within areas of fibrotic (postoperative) scar tissue [14-16, 27]. De Lange et al. analysed the signal intensities of areas suspected for local tumour recurrence on T2-weighted MRI and reported equally low signal intensities for areas of desmoplastic reaction containing tumour and areas of desmoplasia only [27]. It is especially in these cases that the combination of the morphological information from MRI and the functional information from DWI can be beneficial in the diagnosis of recurrent tumour growth. Fibrotic tissue typically has a low cellular density and a large interstitial space, which results in low signal intensity on high b-value (b1000) diffusion images. Conversely, tumoural areas have a relatively high cellular density, which will result in high signal on DWI [28]. Our findings are in concordance with a previous report from Nishie et al, who compared standard MRI to DWI and fusion images for the diagnosis of pelvic tumour recurrences in a group of 28 patients with a variety of primary
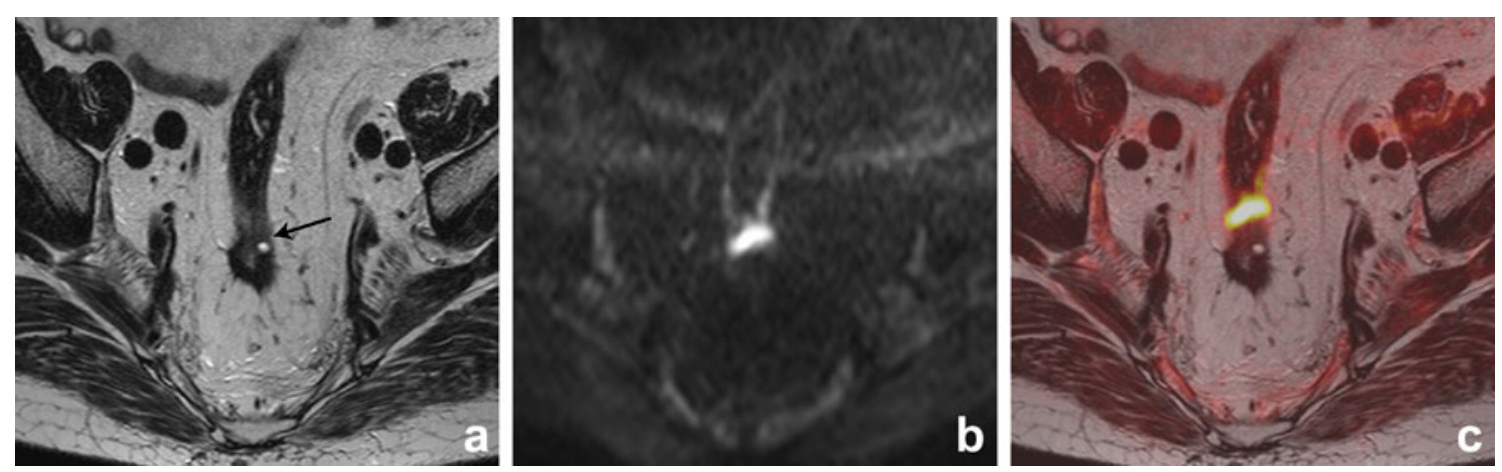

Fig. 4 Axial T2-weighted, b1000 diffusion-weighted and T2W+DWI fusion images of a male patient who had previously undergone transanal endoscopic microsurgery. On T2-weighted MRI (a) there is a small area of intermediate signal intensity (arrow), that was over- looked by reader 2. On the DWI (b) and fusion images (c), there is a clear focal area of high signal intensity and the reader now identified the recurrence, which was later surgically removed and histologically confirmed 
Table 3 Discrepancies between CT findings and MRI

\begin{tabular}{lllll}
\hline Patient $N^{\mathrm{o}}$ & CT findings & \multicolumn{2}{l}{ MRI (+ DWI) } & Outcome (Ref. standard) \\
\cline { 3 - 5 } & & $\mathrm{R} 1$ & $\mathrm{R} 2$ & \\
\hline 2 & & - & - & - \\
5 & Inconclusive & + & + & + \\
12 & Inconclusive & - & - & - \\
14 & - & - & - & + \\
18 & Inconclusive & + & + & - \\
26 & Inconclusive & - & - & + \\
30 & Inconclusive & - & - & + \\
33 & + & + & + & + \\
35 & Inconclusive & + & + & - \\
36 & Inconclusive & + & + & + \\
40 & Inconclusive & - & - & + \\
\hline
\end{tabular}

pelvic malignancies [20]. They found the fusion images of T2-weighted MRI and DWI to perform the best with an AUC of 0.95 . The main gain from the addition of DWI was the improved specificity from $47 \%$ on standard to MRI to $72 \%$ with DWI, indicating that false positivity could be reduced by more than $20 \%$.

As compared to the separate evaluation of the standard MRI and diffusion images, the addition of image fusion did not improve the performance of the readers in our study. Both readers, however, experienced the fusion images to be helpful for a better understanding of the anatomical correlation between standard MRI and DWI. MRI is presently mainly used as a pre-operative roadmap for surgeons to determine the resectability of recurrence in patients who are potential candidates for curative salvage surgery [14]. In this regard, image fusion of standard MRI and DWI might be beneficial and should be addressed by further studies.
In our study, 11 patients had undergone CT imaging prior to MRI. In eight (73\%) cases CT showed equivocal findings and patients had to be referred for further imaging. The readers were not aware of the fact that a CT had been performed previous to the MRI, nor were any of the CT findings revealed. In this study setting, all 8 patients were correctly diagnosed by both readers, both on standard MRI and on MRI plus DWI, suggesting that MRI has superior sensitivity and specificity compared to CT. The limitation of CT remains its inferior soft tissue contrast as compared to MRI. Previous studies that have compared CT and MRI for evaluation of local recurrences have reported similar superiority of MRI, both for the detection of recurrent tumour in this specific group of patients as well as for the assessment of tumour resectability [29, 30]. In the current study, MRI without contrast was used. Some authors have suggested the use of dynamic contrast enhanced (DCE)
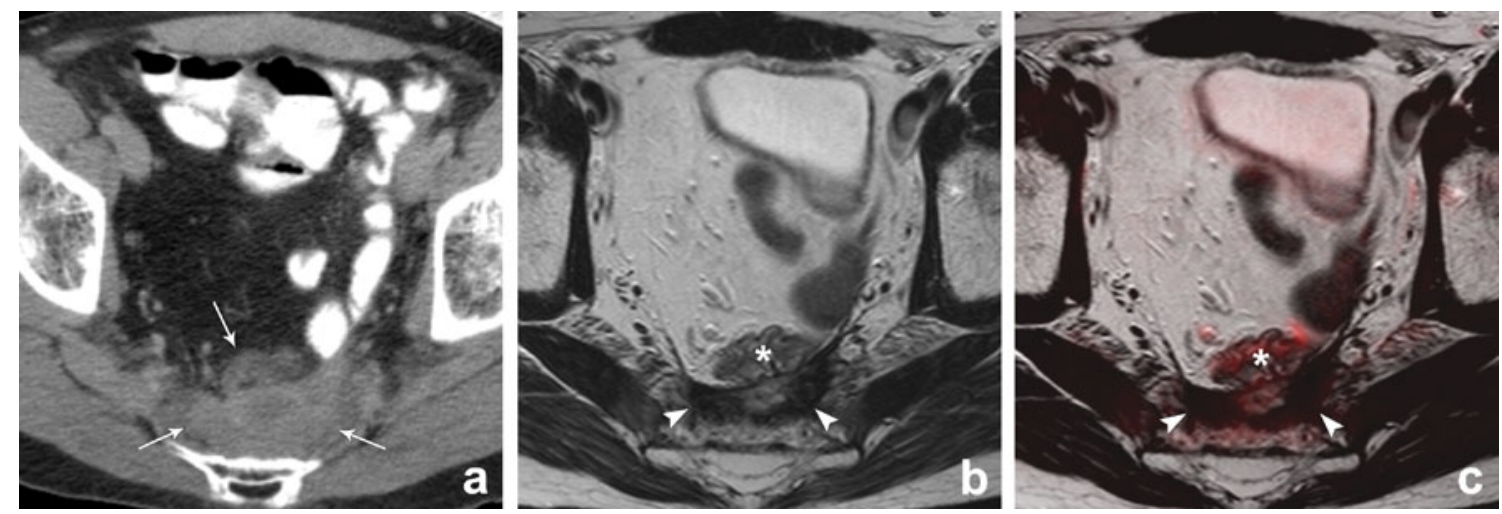

Fig. 5 CT, T2-weighted and T2W+DWI fusion images of a male patient who had previously undergone an abdominoperineal resection for a distally located rectal tumour. On CT (a) there is a presacrally located soft tissue mass. On CT it is not possible to discriminate between postoperative scar tissue and recurrent tumour. On the corresponding MRI (b) there is an area of hypointense signal intensity indicating fibrosis (arrowheads). Located anterior to this fibrosis, there is a bowel loop $\left(^{*}\right)$ This bowel loop was not opacified by the oral contrast on $\mathrm{CT}$, because it was located distally from the patient's ileostomy. On the fusion images (c) there are no areas of high signal intensity, suggestive of recurrent tumour. Follow-up imaging revealed no tumour recurrence 
Gadolinium sequences for a better diagnosis of recurrent tumour [31, 32]. Results are, however, somewhat conflicting as others have shown little improvement for DCE compared to standard morphological MRI [33].

Our study is limited because of its retrospective nature. Furthermore, we only observed a trend towards an improved performance for MRI+DWI, which could partly be due to the small sample size and the relatively high level of experience of both readers in our study. The results of our study are, however, promising and therefore warrant further prospective studies to confirm the value of MRI+DWI in larger patient groups and in a general clinical setting.

In conclusion, this study shows that standard MRI on its own has a high accuracy for the diagnosis of locally recurrent rectal cancer. Addition of DWI does not significantly improve its performance. However, addition of DWI might help radiologists to more confidently rule out the presence of a recurrence. Moreover, the use of DWI can increase interobserver agreement. MRI is therefore promising as a tool to diagnose local recurrences after rectal cancer surgery in the group of patients with a high clinical suspicion for recurrence. Fusion images of MRI+DWI do not further improve the diagnostic accuracy.

Open Access This article is distributed under the terms of the Creative Commons Attribution Noncommercial License which permits any noncommercial use, distribution, and reproduction in any medium, provided the original author(s) and source are credited.

\section{References}

1. Jeffery M, Hickey BE, Hider PN (2007) Follow-up strategies for patients treated for non-metastatic colorectal cancer. Cochrane Database Syst Rev 24:CD002200

2. Kusters M, Marijnen CA, van de Velde CJ, Rutten HJ, Lahaye MJ, Kim JH, Beets-Tan RG, Beets GL (2010) Patterns of local recurrence in rectal cancer; a study of the Dutch TME trial. Eur J Surg Oncol 36:470-476

3. Caricato M, Borzomati D, Ausania F, Valeri S, Rosignoli A, Coppola R (2006) Prognostic factors after surgery for locally recurrent rectal cancer: an overview. Eur J Surg Oncol 32:126132

4. Desch CE, Benson AB 3rd, Somerfield MR, Flynn PJ, Krause C, Loprinzi CL, Minsky BD, Pfister DG, Virgo KS, Petrelli NJ, American Society of Clinical Oncology (2005) Colorectal cancer surveillance: 2005 update of an American society of clinical oncology practice guideline. J Clin Oncol 23:8512-8519

5. Glimelius B, Påhlman L, Cervantes A, ESMO Guidelines Working Group (2010) Rectal cancer: ESMO clinical practice guidelines for diagnosis, treatment and follow-up. Ann Oncol 21 (Suppl 5):v82-86

6. Titu LV, Nicholson AA, Hartley JE, Breen DJ, Monson JR (2006) Routine follow-up by magnetic resonance imaging does not improve detection of resectable local recurrences from colorectal cancer. Ann Surg 243:348-352
7. Schaefer O, Langer M (2007) Detection of recurrent rectal cancer with CT, MRI and PET/CT. Eur Radiol 17:2044-54

8. Valentini V, Aristei C, Glimelius B, Minsky BD, Beets-Tan R, Borras JM, Haustermans K, Maingon P, Overgaard J, Pahlman L, Quirke P, Schmoll HJ, Sebag-Montefiore D, Taylor I, Van Cutsem E, Van de Velde C, Cellini N, Latini P, Committee S (2009) Multidisciplinary rectal cancer management: 2 nd european rectal cancer consensus conference (EURECA-CC2). Radiother Oncol $92: 148-163$

9. Takeuchi O, Saito N, Koda K, Sarashina H, Nakajima N (1999) Clinical assessment of positron emission tomography for the diagnosis of local recurrence in colorectal cancer. Br J Surg 86:932-937

10. Flamen P, Stroobants S, Van Cutsem E, Dupont P, Bormans G, De Vadder N, Penninckx F, Van Hoe L, Mortelmans L (1999) Additional value of whole-body positron emission tomography with fluorine-18-2-fluoro-2-deoxy-D-glucose in recurrent colorectal cancer. J Clin Oncol 17:894-901

11. Even-Sapir E, Parag Y, Lerman H, Gutman M, Levine C, Rabau M, Figer A, Metser U (2004) Detection of recurrence in patients with rectal cancer: PET/CT after abdominoperineal or anterior resection. Radiology 232:815-822

12. Vliegen RF, Beets-Tan RG, Vanhauten B, Driessen A, Oellers M, Kessels AG, Arens A, Beets GL, Buijsen J, van Baardwijk A, de Ruysscher D, Lammering G (2008) Can an FDG-PET/CT predict tumor clearance of the mesorectal fascia after preoperative chemoradiation of locally advanced rectal cancer? Strahlenther Onkol 184:457-464

13. Dresen RC, Kusters M, Daniels-Gooszen AW, Cappendijk VC, Nieuwenhuijzen GA, Kessels AG, de Bruïne AP, Beets GL, Rutten HJ, Beets-Tan RG (2010) Absence of tumor invasion into pelvic structures in locally recurrent rectal cancer: prediction with preoperative MR imaging. Radiology 256:143-150

14. Dresen RC, Beets GL, Rutten HJ, Engelen SM, Lahaye MJ, Vliegen RF, de Bruïne AP, Kessels AG, Lammering G, Beets-Tan RG (2009) Locally advanced rectal cancer: MR imaging for restaging after neoadjuvant radiation therapy with concomitant chemotherapy. Part I. Are we able to predict tumor confined to the rectal wall? Radiology 252:71-80

15. Kuo LJ, Chern MC, Tsou MH, Liu MC, Jian JJ, Chen CM, Chung YL, Fang WT (2005) Interpretation of magnetic resonance imaging for locally advanced rectal carcinoma after preoperative chemoradiation therapy. Dis Colon Rectum 48:23-28

16. Barbaro B, Vitale R, Leccisotti L, Vecchio FM, Santoro L, Valentini V, Coco C, Pacelli F, Crucitti A, Persiani R, Bonomo L (2010) Restaging locally advanced rectal cancer with MR imaging after chemoradiation therapy. Radiographics 30:699-716

17. Koh DM, Collins DJ (2007) Diffusion-weighted MRI in the body: applications and challenges in oncology. AJR Am J Roentgenol 188:1622-1635

18. Namimoto T, Awai K, Nakaura T, Yanaga Y, Hirai T, Yamashita Y (2009) Role of diffusion-weighted imaging in the diagnosis of gynecological diseases. Eur Radiol 19:745-760

19. Rao SX, Zeng MS, Chen CZ, Li RC, Zhang SJ, Xu JM, Hou YY (2008) The value of diffusion-weighted imaging in combination with T2-weighted imaging for rectal cancer detection. Eur J Radiol 65:299-303

20. Nishie A, Stolpen AH, Obuchi M, Kuehn DM, Dagit A, Andresen K (2008) Evaluation of locally recurrent pelvic malignancy: performance of T2- and diffusion-weighted MRI with image fusion. J Magn Reson Imaging 28:705-713

21. Tsushima Y, Takano A, Taketomi-Takahashi A, Endo K (2007) Body diffusion-weighted MR imaging using high b-value for malignant tumor screening: usefulness and necessity of referring to $\mathrm{T} 2$-weighted images and creating fusion images. Acad Radiol $14: 643-650$ 
22. Takahara T, Imai Y, Yamashita T, Yasuda S, Nasu S, Van Cauteren M (2004) Diffusion weighted whole body imaging with background body signal suppression (DWIBS): technical improvement using free breathing, STIR and high resolution 3D display. Radiat Med 22:275-282

23. Rosset A, Spadola L, Ratib O (2004) OsiriX: an open-source software for navigating in multidimensional DICOM images. J Digit Imaging 17:205-216

24. DeLong ER, DeLong DM, Clarke-Pearson DL (1988) Comparing the areas under two or more correlated receiver operating characteristic curves: a nonparametric approach. Biometrics 44:837-845

25. Cohen J (1986) Weighted kappa: nominal scale agreement with provision for scaled disagreement or partial credit. Psychol Bull 70:213-220

26. Syk E, Torkzad MR, Blomqvist L, Nilsson PJ, Glimelius B (2008) Local recurrence in rectal cancer: anatomic localization and effect on radiation target. Int J Radiat Oncol Biol Phys 72:658-664

27. de Lange EE, Fechner RE, Wanebo HJ (1989) Suspected recurrent rectosigmoid carcinoma after abdominoperineal resection: MR imaging and histopathologic findings. Radiology 170:323-328

28. Vandecaveye V, De Keyzer F, Nuyts S, Deraedt K, Dirix P, Hamaekers P, Vander Poorten V, Delaere P, Hermans R (2007)
Detection of head and neck squamous cell carcinoma with diffusion weighted MRI after (chemo)radiotherapy: correlation between radiologic and histopathologic findings. Int J Radiat Oncol Biol Phys 67:960-971

29. Blomqvist L, Holm T, Goranson H, Jacobsson H, Ohlsen $H$, Larsson SA (1996) MR imaging, CT and CEA scintigraphy in the diagnosis of local recurrence of rectal carcinoma. Acta Radiol 37:779-784

30. Beets-Tan RG, Beets GL, Borstlap AC, Oei TK, Teune TM, von Meyenfeldt MF, van Engelshoven JM (2000) Preoperative assessment of local tumor extent in advanced rectal cancer: CT or high-resolution MRI? Abdom Imaging 25:533-541

31. Kinkel K, Tardivon AA, Soyer P, Spatz A, Lasser P, Rougier P, Vanel D (1996) Dynamic contrast-enhanced subtraction versus T2-weighted spin-echo MR imaging in the follow-up of colorectal neoplasm: a prospective study of 41 patients. Radiology 200:453458

32. Torricelli P, Pecchi A, Luppi G, Romagnoli R (2003) Gadoliniumenhanced MRI with dynamic evaluation in diagnosing the local recurrence of rectal cancer. Abdom Imaging 28:19-27

33. Blomqvist L, Fransson P, Hindmarsh T (1998) The pelvis after surgery and radio-chemotherapy for rectal cancer studied with $\mathrm{Gd}-$ DTPA-enhanced fast dynamic MR imaging. Eur Radiol 8:781-787 